\title{
General Coastline Extraction Based on an Improved Active Contour Model in Jiaozhou Bay, Qingdao, China, from 1990 to 2018 Using Landsat Satellite Images
}

\author{
Jie Dong $\mathbb{D}^{1,2}$ Jiani Fu, ${ }^{1,2}$ Yong Guan, ${ }^{1,2}$ Haisong Liu, ${ }^{1,2}$ Qing Wang, ${ }^{1,2}$ and Ming Hao $\mathbb{D}^{3}$ \\ ${ }^{1}$ Qingdao Geo-Engineering Surveying Institute (Qingdao Geological Exploration Development Bureau), Qingdao 266071, China \\ ${ }^{2}$ Key Laboratory of Geological Safety of Coastal Urban Underground Space, Ministry of Natural Resources, Qingdao 266071, China \\ ${ }^{3}$ Jiangsu Key Laboratory of Resources and Environmental Information Engineering, China University of Mining and Technology, \\ Xuzhou 221116, China
}

Correspondence should be addressed to Jie Dong; 13853286966@163.com

Received 8 July 2021; Revised 23 August 2021; Accepted 6 October 2021; Published 31 October 2021

Academic Editor: Alberto Alvarez-Gallegos

Copyright (c) 2021 Jie Dong et al. This is an open access article distributed under the Creative Commons Attribution License, which permits unrestricted use, distribution, and reproduction in any medium, provided the original work is properly cited.

The coastline is located at the junction of the sea and the land, and it is essential for ecological environment. However, most existing methods can extract the coastline with obvious boundaries and cannot obtain the general coastline, including an intertidal zone and salt field. Accordingly, a new general coastline extraction method is proposed on the basis of an improved active contour model to extract the general coastline from remote sensing images. An improved active contour model was proposed to extract the water area by introducing aiming energy of water from the Modified Normalized Difference Water Index information. Then, mathematical morphology was applied to obtain the seawater area based on the extracted water area. Finally, the coastline was refined and generated by the improved active contour model in a buffer zone of the seawater boundary. Landsat images over Jiaozhou Bay in Shandong Province, China, from 1990 to 2018 were used to extract the general coastline. Results demonstrate that the proposed method can effectively extract the general coastline, which is close to the reference coastline. The length of the coastline decreased from $234.64 \mathrm{~km}$ in 1990 to $221.21 \mathrm{~km}$ in 2000 . This value significantly increased to $255.05 \mathrm{~km}$ from 2000 to 2010 . The main reason is that Hongdao Island merged with the mainland due to reclamation. The length of the coastline slightly decreased by approximately $12 \mathrm{~km}$ from 2010 to 2018 due to environmental protection measures and the reclamation prohibition.

\section{Introduction}

The coastline is located at the junction of the sea and the land. It has become an important area for human development due to its unique geographical characteristics. The coastline is constantly expanding or contracting with complexity and variability due to the influence of coast swell, erosion, climate warming, artificial dam construction, and landfills in the coastal zone. Therefore, coastline detection is one of the key concerns of the environmental department.

At present, two main methods for coastline detection are available. The first one is manual visual interpretation [1]. This method uses a manual approach of describing the boundary between the land and the sea based on the com- parison of colour information, texture information, and coastline trend information on remote sensing images. However, this method is time consuming and laborious and requires extensive experience for visual interpretation. The other method is automatic computer interpretation [2]. The shortcoming of manual visual interpretation can be overcome by using the remote sensing technology, which has the advantages of large-area imaging and real-time processing [3]. Considering the characteristics of the sea and land boundaries, the extraction of the waterline is essentially the process of remote sensing image segmentation. The image is divided into land and sea areas. The intersection of the two areas is the instantaneous waterline. Segmentation methods include threshold segmentation $[4,5]$, region 
growing [6], edge detection [7], and neural network [8, 9]. However, most existing methods may not be able to extract the existing aquaculture coastline along the coast. Active contour models (ACMs) [10], which are one of the most advanced coastline extraction techniques, are flexible because they can construct energy functions to deal with complex coastal conditions. These models have the advantages of implicit handling of topological changes and low sensitivity to noise [11] and can be used to detect objects without defined boundaries by gradient. Zhu et al. [12] proposed a coastline extraction method by integrating prior edge information with an active contour model based on multispectral images. The proposed method indicated the advantage of aquaculture coastline extraction of an active contour model. According to the basic information contained in the energy pan function, active contour models can be divided into edge and regional active contour models.

The edge active contour model mainly uses the gradient information of the image edge, including the snake model [10] and geodesic active contour model [13]. The snake model cannot generally extract the position information of the concave edge. The construction of a geodesic active contour model is based on the principle that the "ray" in optics determines the path according to the shortest optical path [13]. The edge-based active contour model only uses the edge features of the image. When the edge is obscured in the image, the method will be restricted, and the ideal segmentation effect cannot be obtained.

The regional active contour model uses the overall greyscale information of the image area. The model starts with the Mumford-Shah model, but it is difficult to solve. Accordingly, the Chan-Vese (CV) model simplified the Mumford-Shah model and introduced the level set function to make it more convenient and natural to deal with the topological changes of the curve [11]. Zhu et al. proposed a new method that integrates prior edge information with an edge-based active contour model [12]. The coarse sea region is obtained with a novel region growth method, and an edge refining process is proposed by integrating an edge-based active contour model for the prior edge information. Finally, the coastline is obtained via contour evolution. Shi et al. proposed a coastline extraction algorithm by utilising superpixels as the basic unit [14]. An improved line finder was used in the simple linear iterative clustering to enhance results around the dam. A Gabor feature and hidden Markov random field model were utilized to classify superpixels to generate a coastline. However, most existing methods can extract the coastline with obvious boundaries and cannot obtain the general coastline, including an intertidal zone and salt field.

A general coastline extraction method based on an improved active contour model is proposed in this work. The proposed method consists of three main steps, namely, the Modified Normalized Difference Water Index (MNDWI) information production, water area extraction using the improved active contour, and the general coastline extraction, as shown in Figure 1. Firstly, MNDWI was applied to images to obtain water information based on the green and middle-infrared band information. Secondly, the improved active contour model was used to extract the coarse water area. Thirdly, the coarse water areas were processed by mathematical morphology to generate the seawater area, and the general coastline was then extracted. Finally, the coastline was refined to remove the influence of mathematical morphological processing. Three Landsat 5 Thematic Mapper (TM) images obtained in 1990, 2000, and 2010 and one Landsat 8 Operational Land Imager (OLI) image acquired in 2018 were examined to evaluate the proposed method.

\section{Materials and Methods}

2.1. Study Area. Jiaozhou Bay is a semiclosed bay located in the middle of the Yellow Sea in Shandong Province, China, as shown in Figure 2. The total area of this region is approximately $500 \mathrm{~km}^{2}\left(36^{\circ} 00^{\prime}-36^{\circ} 20^{\prime} \mathrm{N}, 120^{\circ} 00^{\prime}-120^{\circ} 30^{\prime} \mathrm{E}\right)$, and the administrative regions along the coast of Jiaozhou Bay are Shinan District, Shibei District, Licang District, Chengyang District, Jiaozhou City, and Huangdao District in Qingdao since 2012. The study area is in a mediumlatitude monsoon environment. The average annual precipitation is approximately $662.1 \mathrm{~mm}$, and precipitation is more concentrated in summer. Jiaozhou Bay is located in the urban area of Qingdao, and it looks like a bell mouth; hence, its coastline is vast and has a complex form, making it difficult to extract. Jiaozhou Bay is a regular half-day tide level. Qingdao's average sea level is $2.42 \mathrm{~m}$, controlled by the high tide level of Dagang, with an average high tide level of $3.81 \mathrm{~m}$, an average low tide level of $1.02 \mathrm{~m}$, and an average tidal range of $2.78 \mathrm{~m}$.

2.2. Experimental Data. Landsat images have been used to study how the Earth's surface has been changing for decades due to its long history and relatively high spatial resolution. Many studies used time series Landsat images due to their long data collection time, large storage capacity, and free access to Landsat data, and some of them were focused on coastline extraction.

This study selects Landsat images from 1990 to 2018 (i.e., 1990, 2000, 2010, and 2018) to perform coastline extraction and time series analysis. The information of used images in this study is shown in Table 1 and Figure 3. The imaging time of the Landsat remote sensing satellite in Qingdao was approximately 10:36 am (Beijing time). According to the tide table of Jiaozhou Bay, the tide was at a low level when the sensors were imaging on May 24, 1990, May 19, 2000, and October 12, 2018. Meanwhile, the tide was at a high level when the sensors were imaging on October 6, 2010. Nonetheless, the tide heights in the four imaging times were approximately $1 \mathrm{~m}$. Therefore, the tide has a slight effect on the coastline extraction in this study.

The reference coastline was used to evaluate the effectiveness of the proposed method, which included natural coastline, artificial coastline, and other coastlines. Natural shoreline includes bedrock shoreline, sandy shoreline, and silty muddy shoreline; artificial shoreline is a shoreline composed of permanent artificial structures, and other coastlines include estuarine coastlines and coastlines with natural 


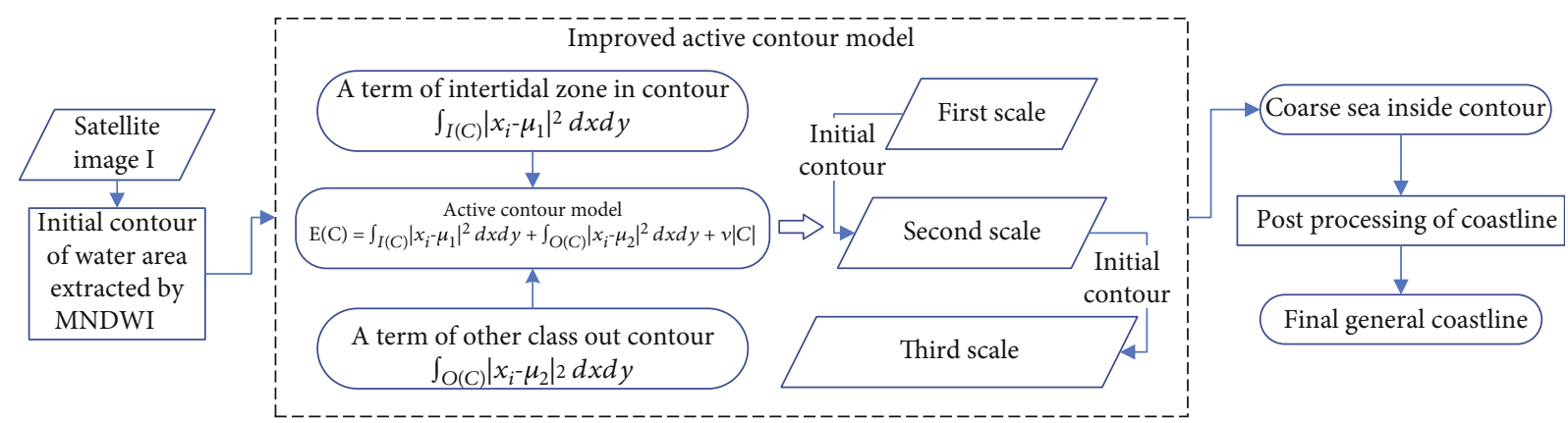

FigURE 1: Flowchart of the proposed general coastline extraction method.

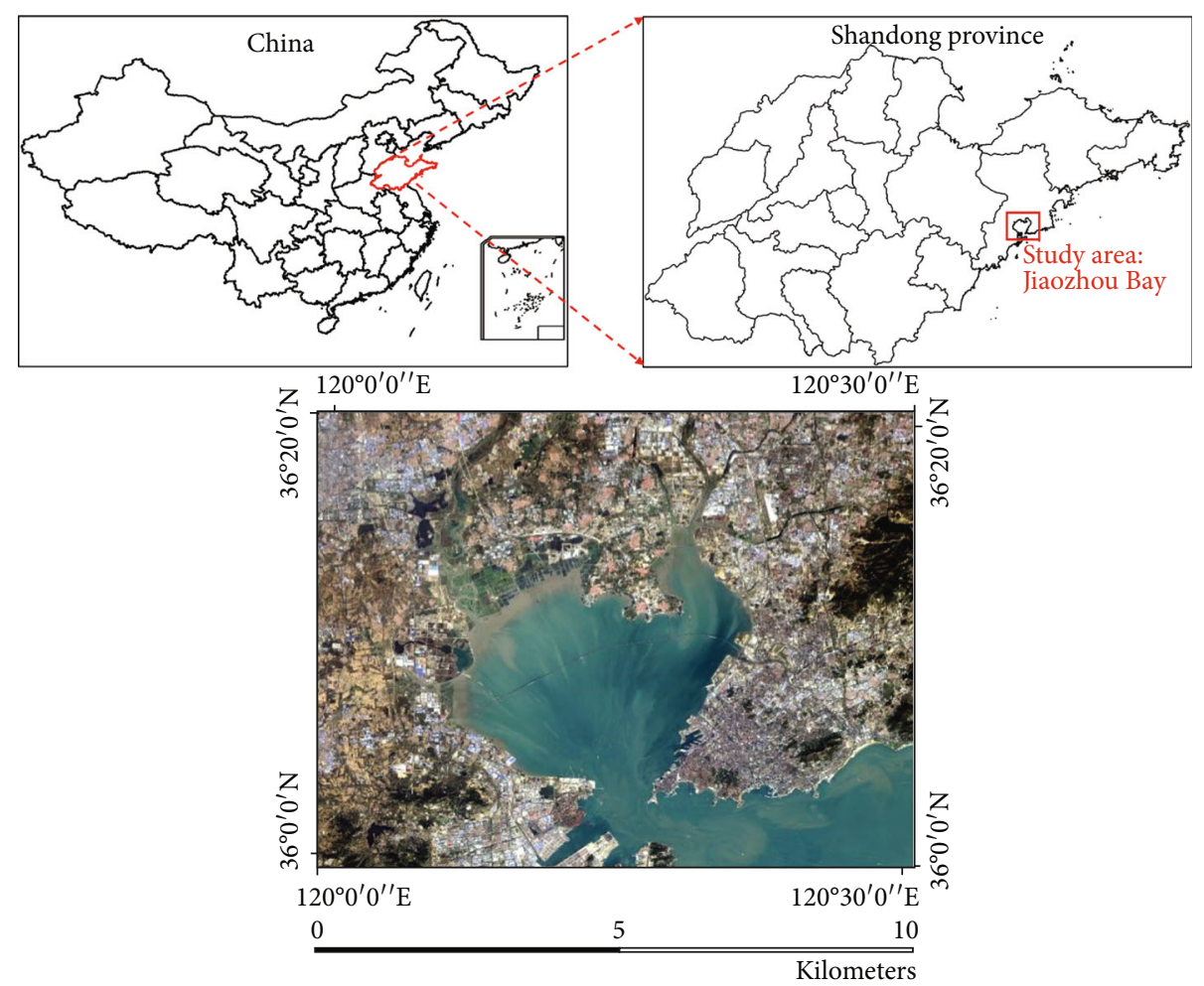

FIgURE 2: Location of the study area: (a) Landsat 8 remote sensing image of Jiaozhou Bay.

TABLE 1: Information of used images in this study.

\begin{tabular}{lcc}
\hline Data & Satellites and sensors & Spatial resolution $(\mathrm{m})$ \\
\hline 1990.05 .24 & Landsat 5 TM & 30 \\
2000.05 .19 & Landsat 5 TM & 30 \\
2010.10 .06 & Landsat 5 TM & 30 \\
2018.10 .12 & Landsat 8 OLI & 30 \\
\hline
\end{tabular}

beach morphology and ecological functions. The reference coastline was obtained through visual interpretation, but it is not public data at present.

\subsection{Methods}

2.3.1. Initial Contour of the Water Area Extracted by MNDWI. The MNDWI index is adopted to enhance water information based on the comparison between different algo- rithms, such as NDVI, MNDWI, spectral angle mapper, and automated water extraction index [15]. The aforementioned index is calculated from the Landsat images as follows [4]:

$$
\text { MNDWI }=\frac{\text { Green }- \text { MIR }}{\text { Green }+ \text { MIR }}
$$

where Green and MIR are the image reflectance on the green and middle-infrared bands, respectively.

Water bodies are expected to yield a positive MNDWI, while land surfaces should be negative. MNDWI was applied to the remote sensing image acquired in 1990, and the result and its histogram are shown in Figure 4 . The result showed that water bodies are clearly distinguished from other ground features. OTSU [16] was applied to the MNDWI layer to extract the water area and automatically determine a water classification threshold. Finally, the extracted water 


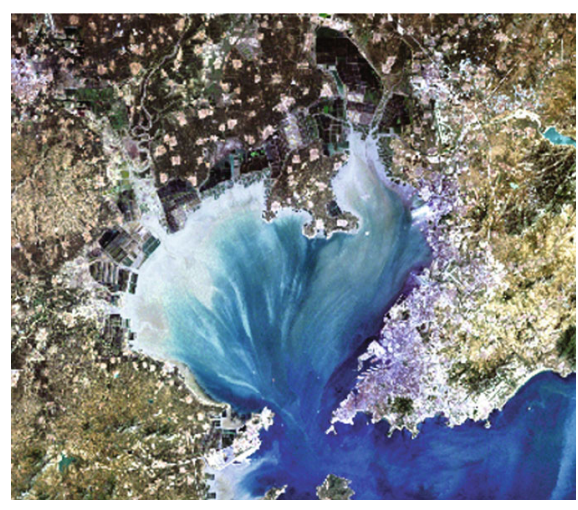

(a)

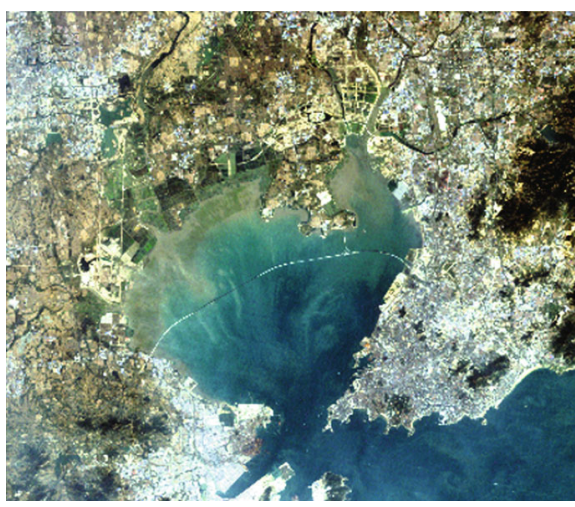

(c)

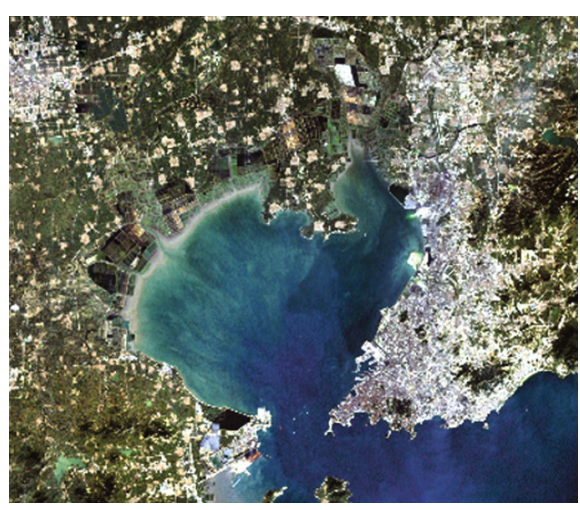

(b)

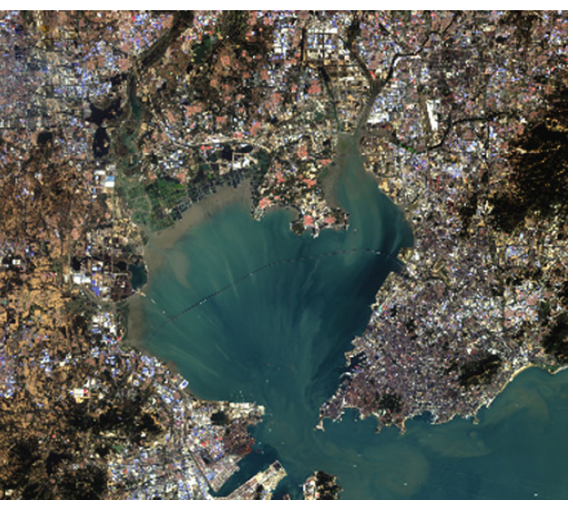

(d)

FIGURE 3: Landsat data schematic map of true colour data: (a) 1990.05.24, (b) 2000.05.19, (c) 2010.10.06, and (d) 2018.10 .12$.

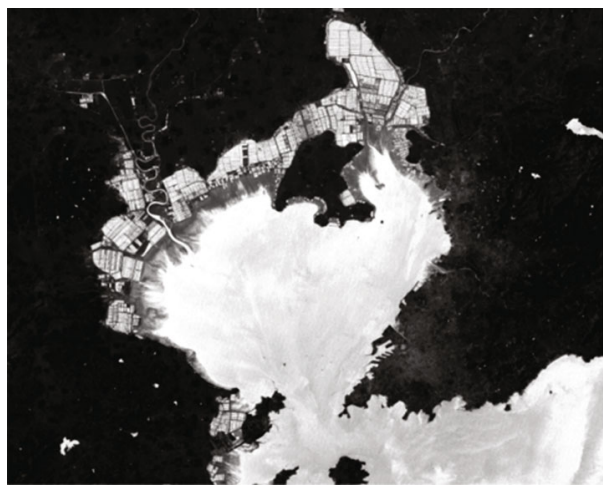

(a)

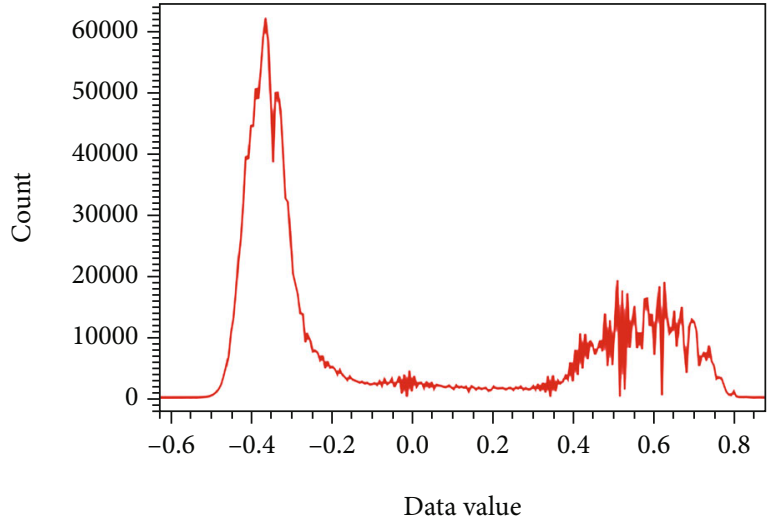

(b)

FIGURE 4: MNDWI image and its histogram of the remote sensing image acquired in 1990: (a) MNDWI image and (b) MNDWI histogram.

area was produced to initial values in the following active contour model.

2.3.2. Improved Active Contour Model. The coastline extraction can be achieved by finding an optimal contour $C$ by the energy functional given as follows [11]:

$$
E(C)=\int_{I(C)}\left|x_{i}-c_{1}\right|^{2} d x d y+\int_{O(C)}\left|x_{i}-c_{2}\right|^{2} d x d y+v|C|,
$$

where $v>0$ is a constant, $C$ is the evolving contour, $I(C)$ represents the area inside the evolving contour and can be seen as changed, $O(C)$ denotes the area outside the evolving contour and can be regarded as unchanged, $|C|$ denotes the length of the evolving contour, $x_{i}$ is the $i$ th pixel value of the difference image, and $c_{1}$ and $c_{2}$ are the grey average of all pixels inside and outside the contour $C$, respectively. The term $\int_{I(C)}\left|x_{i}-c_{1}\right|^{2} d x d y$ is a sum of the differences between pixel grey values inside the contour $C$ and their corresponding 
mean values. Meanwhile, the term $\int_{O(C)}\left|x_{i}-c_{2}\right|^{2} d x d y$ is a sum of the differences between the pixel grey values outside the contour $C$ and their corresponding mean values. The contour then evolves to fit the edges of objects by making equation (2) reach a minimum value. Finally, the image is segmented into changed and unchanged parts, each with homogeneous pixels.

Two new terms are added into equation (2) to eliminate the need for initial contours and improve the accuracy of change detection with the level set. This aspect refers to the differences between the pixels of the different images and the means of changed and unchanged pixels estimated by the expectation-maximisation algorithm [11]. The new equation can be written as $[17,18]$

$$
\begin{aligned}
E(C)= & \int_{I(C)}\left|x_{i}-c_{1}\right|^{2} d x d y+\int_{O(C)}\left|x_{i}-c_{2}\right|^{2} d x d y+v|C| \\
& +\int_{I(C)}\left|x_{i}-\mu_{1}\right|^{2} d x d y+\int_{O(C)}\left|x_{i}-\mu_{2}\right|^{2} d x d y,
\end{aligned}
$$

where $\mu_{1}$ and $\mu_{2}$ are the mean grey values of the intertidal zone and land in the satellite image, respectively. The term $\int_{I(C)}\left|x_{i}-c_{1}\right|^{2} d x d y$ means a sum of the differences between the pixel grey values inside the contour $C$ and the mean values of the intertidal zone pixels. Meanwhile, the term $\int_{O(C)}\left|x_{i}-c_{2}\right|^{2} d x d y$ is a sum of the differences between the pixel grey values outside the contour $C$ and the mean value of the land pixels. The two terms can ensure that the changed and unchanged pixels are correctly detected without initial contours and improve the distinguishability between the changed and unchanged pixels. These two values can be obtained by the extracted water area by OTSU in the last step.

The level set method is exploited to minimize energy equation (3) [19]. In the level set method, $\Omega$ represents the image domain, and the curve $C$ is represented by the zerolevel set of Lipchitz function $\varphi$, such that

$$
\begin{cases}\varphi(x, y)>0 & (x, y) \in I(C), \\ \varphi(x, y)=0 & (x, y) \in C, \\ \varphi(x, y)<0 & (x, y) \in O(C) .\end{cases}
$$

Heaviside step function $H$ and Dirac delta function $\delta$ are then introduced to describe the functional as follows:

$$
H(z)=\left\{\begin{array}{ll}
1, & z \geq 0, \\
0, & z<0,
\end{array} \delta(z)=\frac{d}{d z} H(z)\right.
$$

The energy function can be described using level set function $\varphi$ instead of unknown variable C. Equation (3) can be written as follows:

$$
\begin{aligned}
E(\varphi)= & \int_{\Omega}\left|x_{i}-c_{1}\right|^{2} H(\varphi) d x d y+\int_{\Omega}\left|x_{i}-c_{2}\right|^{2}(1-H(\varphi)) d x d y \\
& +v \int_{\Omega} \delta(\varphi)|\nabla \varphi| d x d y+\int_{\Omega}\left|x_{i}-\mu_{1}\right|^{2} H(\varphi) d x d y \\
& +\int_{\Omega}\left|x_{i}-\mu_{2}\right|^{2}(1-H(\varphi)) d x d y .
\end{aligned}
$$

where $\nabla \varphi$ means the gradient of $\varphi$. Functions $H$ and $\delta$ are replaced by the regularised versions to obtain a global minimiser:

$$
H_{\varepsilon}(z)=\frac{1}{2}\left(1+\frac{2}{\pi} \arctan \left(\frac{z}{\varepsilon}\right)\right), \delta_{\varepsilon}(z)=\frac{1}{\pi} \frac{\varepsilon}{\varepsilon^{2}+z^{2}} .
$$

Based on the above, the next steps are iteratively performed until the optimal $\varphi$ is obtained [11]. The energy functional is minimised with respect to $c_{1}$ and $c_{2}$ by keeping $\varphi$ fixed, and they can be obtained by the following equations:

$$
c_{1}=\frac{\int_{\Omega} x_{i} H(\varphi) d x d y}{\int_{\Omega} H(\varphi) d x d y}, c_{2}=\frac{\int_{\Omega} x_{i}(1-H(\varphi)) d x d y}{\int_{\Omega}(1-H(\varphi)) d x d y} .
$$

The energy functional is minimised with respect to $\varphi$ by keeping $c_{1}$ and $c_{2}$ fixed. The associated Euler-Lagrange equation parameterising the descent direction by an artificial time $t(t \geq 0)$ is provided as follows:

$$
\frac{\partial \varphi}{\partial t}=\delta_{\varepsilon}(\varphi)\left[v \operatorname{div}\left(\frac{\nabla \varphi}{|\nabla \varphi|}\right)-\left|x_{i}-c_{1}\right|^{2}+\left|x_{i}-c_{2}\right|^{2}-\left|x_{i}-\mu_{1}\right|^{2}+\left|x_{i}-\mu_{2}\right|^{2}\right] .
$$

A finite difference implicit scheme is then used to discretise the equation and achieve the solution of the evolution equation in $\varphi$. Chan and Vese provided further details about numerical approximation [11]. The extraction procedure by ACM is summarised as follows [20]:

Step 1. Initialise $\varphi^{k}$ by $\varphi^{0}$, with $\varphi^{k}$ defined as the distance function from an initial curve $C$ at the $k$ th time iteration $(k=0)$.

Step 2. Compute the average values of $c_{1}^{k}$ and $c_{2}^{k}$ for $\varphi^{k}>0$ and $\varphi^{k} \leq 0$, respectively.

Step 3. Compute $\varphi^{k+1}$ by solving the following:

$$
\varphi^{k+1}=\varphi^{k}-\Delta t \frac{\partial E}{\partial \varphi}\left(\varphi^{k-1}, c_{1}^{k}, c_{2}^{k}\right)
$$

where $\Delta t$ is the time step.

Step 4. Reinitialise $\varphi$ locally to the signed distance function to the curve. 
Step 5. Check if the convergence is reached. Otherwise, $k=$ $k+1$, and go to Step 2 .

The initial contour of the improved ACM of the image acquired in 1990 is shown in Figure 5(a), and the extracted water area is illustrated in Figure 5(b). The water area can be well extracted, including aquaculture coastline in the north of Jiaozhou Bay.

\subsubsection{Postprocessing of Coastline}

(1) Extraction of the General Sea Area Using Mathematical Morphology. Erosion and dilation [21,22] are the most basic forms of computation in binary morphology. In binary morphology, the "probe" (the structural element that determines the shape) is used to detect the image, and the area where the probe can be stored is determined by performing a sounding operation on each set of regions in the image. The structural element is significant for erosion and dilation. The essence of structural elements is a $3 \times 3$ matrix. When a shape has all the structural elements, the centre of the structural element produces an eroded shape. Meanwhile, when only the centre of the pointer is required to reside in the shape, the structure of the dilation section is formed by the child elements of the structural element.

Dilation was implemented to the extracted water area by using the improved CV model with a $9 \times 9$ structural element. Accordingly, the general sea area was connected into a whole area, as shown in Figure 6(a), which was extracted by selecting the largest water area. Other water areas were removed as inland water. The boundary tracing algorithm was used to detect the boundary of a general sea area, namely, the general coastline, as shown in Figure 6(b).

(2) Optimisation of the Extracted General Coastline. The extracted coastline is not completely accurate due to the mathematical morphology processing; therefore, the extracted coastline needs to be further optimised. Firstly, the extracted coastline is used to construct a buffer (as shown in Figure 7(a)). The distance of the buffer is recommended to be 30 pixels in this study considering the intertidal zone. Then, the part of the seawater except for the buffer zone was assigned to the average value of the water body in MNDWI to eliminate the influence of beaches and islands, and the improved active contour model is used to extract the coastline again. The result is shown in Figure 7(b). Finally, the optimised coastline is manually edited in a particularly complex area to generate the final coastline.

\section{Results and Analysis}

3.1. Accuracy of Coastline Extraction and Analysis of Coastline Changes. The proposed method was implemented to the four images and extracted coastlines. In this study, the parameter $v$ in ACM was set manually and empirically to 0.8 , and the number of iterations was set to 100 .
The extracted coastlines are shown in Figure 8. With regard to the 1990, 2000, and 2010 data, the results extracted by the proposed method are close to the reference coastline. The extracted coastline in the east coast with an obvious boundary with the city is basically consistent with the reference data. The extraction results for the northern coast with a large number of salt fields and aquaculture ponds are similar to the reference coastline. The extracted results for the 2018 data have good consistency with the reference coastline on the eastern coast because of the artificially constructed coastline with obvious boundaries. The extracted coastline in the western and northern coasts is generally close to the reference data, but not particularly accurate. The main reason is that the west and the north are the entrances of the Yang and Dagu Rivers, respectively. The complicated river conditions affect the accuracy of the extraction results. In the MNDWI images, towns and beaches are relatively similar due to the large noise of the data in 2018, which also has a certain influence on the extraction results.

The evaluation indices of edge precision (EP) and recall (ER) were employed to validate the effectiveness of the proposed method, which combines image segmentation, region growing, and edge detection together. Two indices with test distance $d$ [12] used for the accuracy assessment of coastline extraction can be obtained as follows:

$$
\begin{aligned}
& \mathrm{EP}=\frac{\mathrm{TP}}{T_{\mathrm{ex}}}, \\
& \mathrm{ER}=\frac{\mathrm{TP}}{T_{\mathrm{tr}}},
\end{aligned}
$$

where TP means the number of true coastline pixels on the extracted coastline, $T_{\mathrm{ex}}$ is the total number of the pixels of the extracted coastline, and $T_{\operatorname{tr}}$ is the total number of pixels of the reference coastline. Extracted coastlines were quantitatively validated using the two indices according to the reference coastlines, as shown in Table 2.

In the 1990, 2000, and 2010 data, the values of EP and ER are greater than 0.7 when $d$ is 1 ; meanwhile, the values of EP and ER are greater than 0.8 when $d$ is 2, indicating that the extracted coastline is close to the reference coastline. In the 2018 data, the precision index values are approximately 0.7 and 0.75 when $d$ is equal to 1 and 2, respectively, which are slightly lower than the results of the above three images. This situation is mainly caused by the complex terrain at the estuary of the Yanghe and Dagu Rivers, and the image data are considerably noisy.

The coastline results of the four years were superimposed, as shown in Figure 9, and the changes in the coastline distribution were analysed. Obvious differences in the southwestern Huangdao Island area were observed from 1990 to 2000 , which covered part of the water area to construct artificial wetlands, such as breeding ponds or reclaiming land. The northern region had expanded to a certain extent due to breeding ponds and salt pan. In the east, the artificial coastline replaced the natural coastline due to the construction of the Jiaozhou Bay Expressway. After 2000, most salt pan and breeding ponds were occupied because of the 


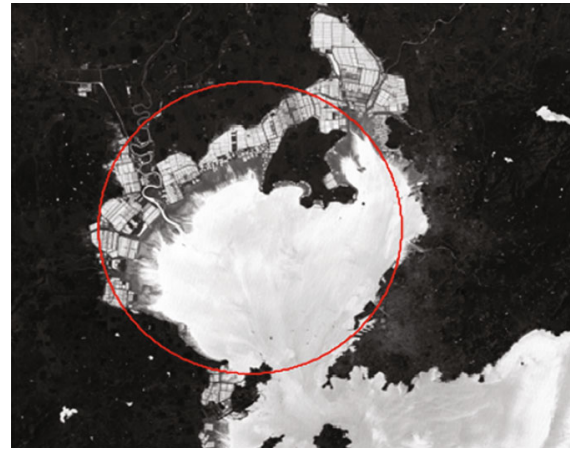

(a)

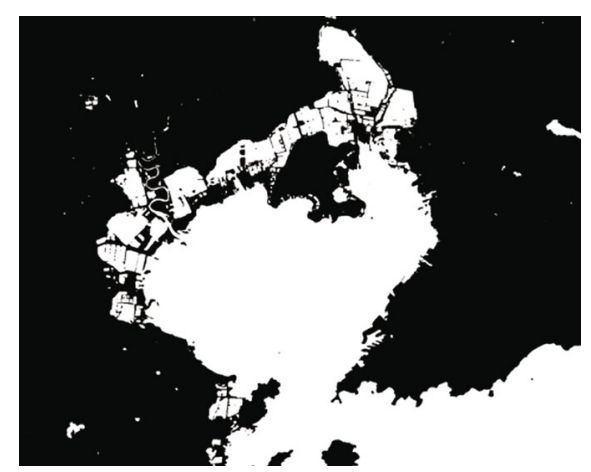

(b)

Figure 5: (a) Initial contour of the improved CV model and (b) extracted water area.

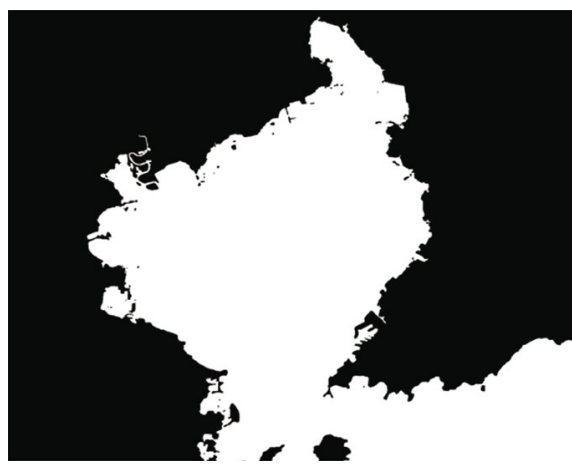

(a)

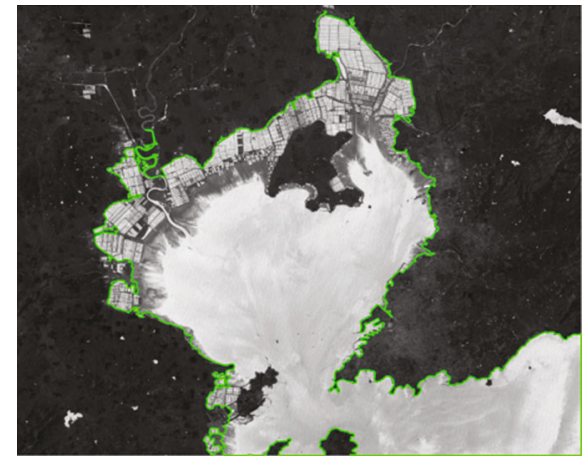

(b)

Figure 6: (a) General sea area extracted by mathematical morphology and (b) general coastline produced by edge tracing.

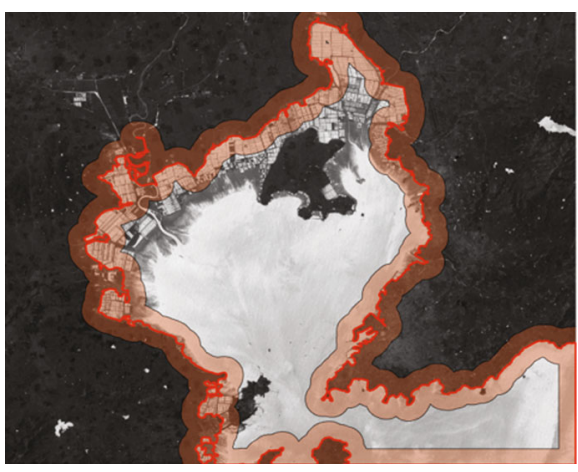

(a)

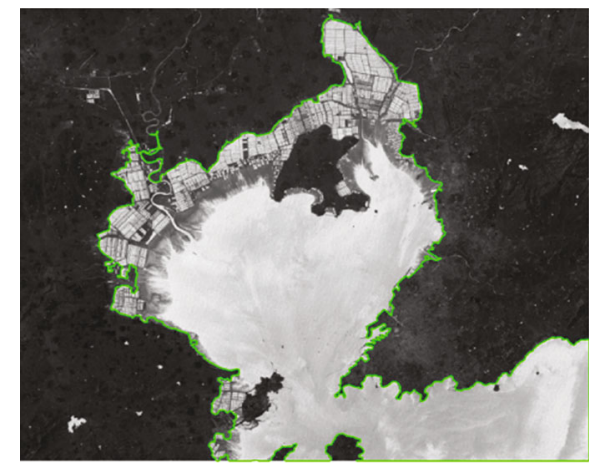

(b)

Figure 7: Optimisation of the general coastline: (a) buffer c5onstructed based on the coastline and (b) optimised coastline.

complete integration of the Hongdao Island and the mainland, and many lands were developed for urban use. Accordingly, the northern coastline of Jiaozhou Bay expanded along the Hongdao Island area, and the artificial characteristics of the eastern coastline became increasingly evident. Barrier dams and breakwaters have increased. The construction of ports in the Huangdao Island area has accelerated, and a port-like artificial coastline has appeared in the southwest.

The changes in the length of the coastline in the past 30 years are shown in Figure 10. The figure also shows that the total length of the coastline has been increasing in recent years (from $234.64 \mathrm{~km}$ in 1990 to $243.01 \mathrm{~km}$ in 2018). The length of the coastline was reduced by $13.43 \mathrm{~km}$ from 1990 to 2000. The coastline length has significantly increased from 2000 to 2010 (about $33.84 \mathrm{~km}$ ). This phenomenon mainly occurred because Hongdao Island was integrated with the mainland. The length of the coastline between 2010 and 2018 decreased by $12.04 \mathrm{~km}$. The length of the coastline decreased because the government began to protect the environment and prohibit reclamation to prevent further expansion of the coastline. 


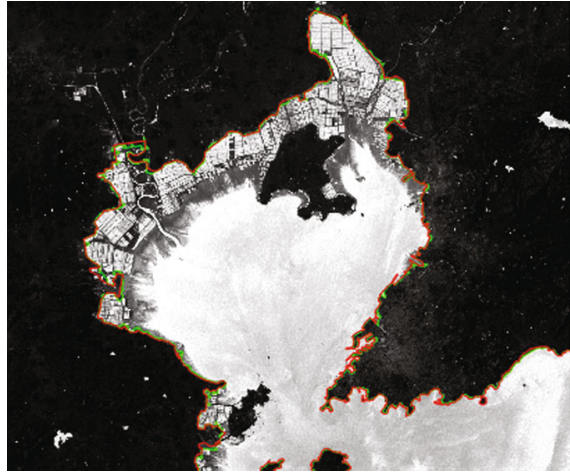

(a)

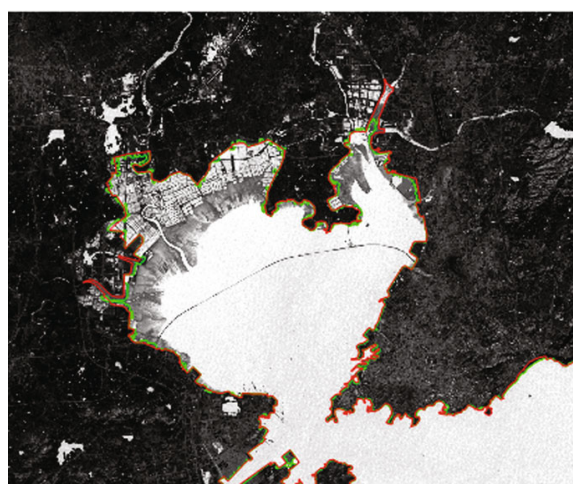

(c)

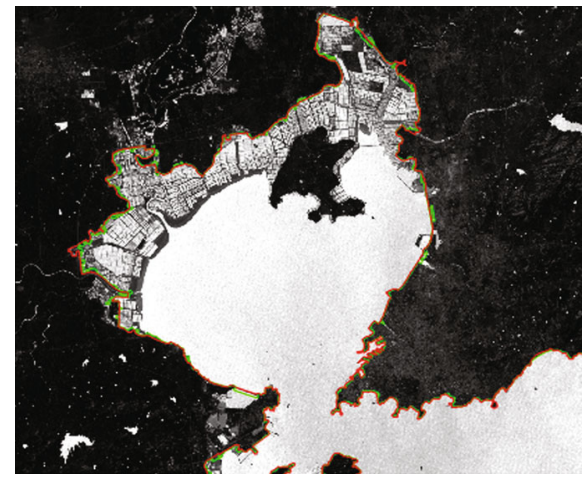

(b)

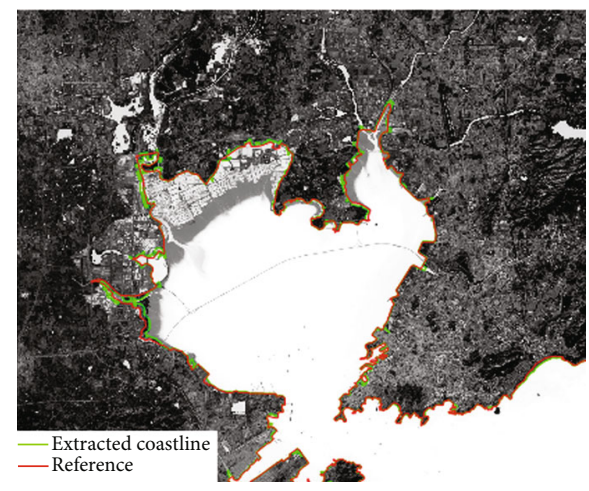

(d)

FIGURE 8: Extracted coastline and corresponding reference coastline of (a) 1990, (b) 2000, (c) 2010, and (d) 2018.

TABLe 2: Accuracy of coastline extraction.

\begin{tabular}{lcccc}
\hline Data & EP & ER & EP & ER \\
\hline 1990.05 .24 & 0.75 & 0.80 & 0.82 & 0.87 \\
2000.05 .19 & 0.75 & 0.81 & 0.83 & 0.88 \\
2010.10 .06 & 0.72 & 0.74 & 0.80 & 0.81 \\
2018.10 .12 & 0.66 & 0.73 & 0.73 & 0.77 \\
\hline
\end{tabular}

\subsection{Factors Caused Coastline Changes}

3.2.1. Natural Factors. The main natural factors that affect the coastline change of Jiaozhou Bay are erosion and siltation change. According to the analysis of historical data, the natural siltation of Jiaozhou Bay mainly comes from two aspects: the first one is the sediment and dust caused by rivers and wind and the other one is the natural sediments affected by the tidal current.

In terms of river sediment deposition, many river systems are along the coast of Jiaozhou Bay, with more than a dozen rivers injected. Some large ones are the Yang, Nanjiaolai, Dagu, and Baisha Rivers. In addition, many unknown gullies are observed, especially on the north side of Jiaozhou Bay, and they radially merge into the bay. Precipitation greatly varies with the seasons because this region is in the monsoon region. There are many intermittent and rain-sourced rivers. The riverbed sediments of these rivers are exposed during the dry season. Meanwhile, most of the sediment will be merged into the Jiaozhou Bay by the river during the wet season. Consequently, the coastal waters on the northeast and northwest sides of the Jiaozhou Bay will silt up, causing the coastline to move forward.

Tidal current deposition is characterised into two aspects. On the one hand, it is related to the tidal current characteristics of Jiaozhou Bay. The rising tide speed is faster than the falling tide speed, and the rising tide lasts shorter than the falling tide. The change in the speed of the ups and downs of the tidal current causes the larger particles carried by the tidal current to deposit in the Jiaozhou Bay, and the sediment is difficult to carry out of the bay. On the other hand, it is also related to the geographical shape of Jiaozhou Bay. Jiaozhou Bay is an intrusive water area of the Yellow Sea to the mainland. The bay mouth is narrow, and the bay is wide. As the tidal water enters the open water from the narrow bay mouth, the wave energy diverges, and the offshore sediment deposits.

3.2.2. Human Factors. The human activities along the Jiaozhou Bay are mainly manifested in reclamation, land reclamation, and municipal waste dumping. The impact of human activities has also converted most coasts of Jiaozhou Bay from the natural coasts to the artificial coasts.

Reclamation mainly occurs in the western, northern, and northeastern regions. Hongdao Island and Huangdao Island were not connected to land in the past; however, they have become part of the mainland due to reclamation. A large number of dams were built to prevent the impact of waves on the salt pan and breeding ponds. These projects cause 


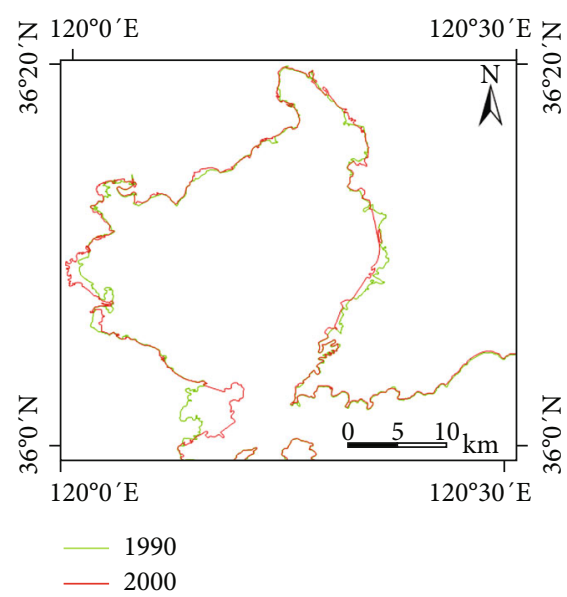

(a)

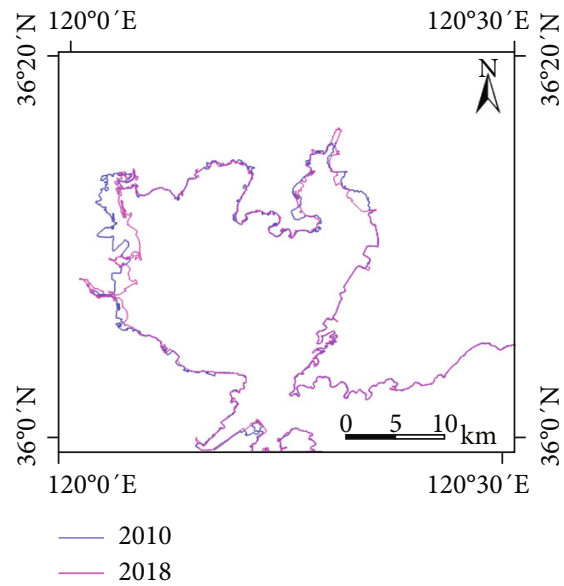

(c)

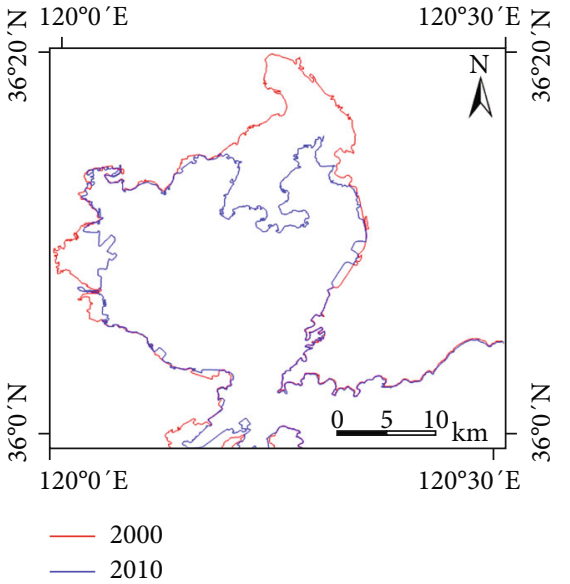

(b)

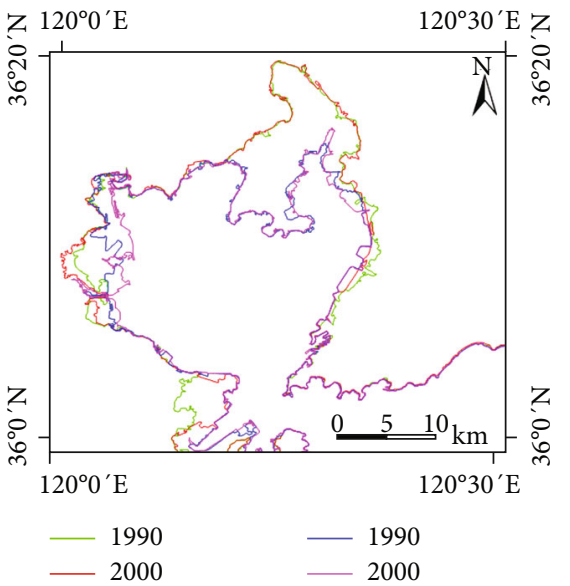

(d)

FIGURE 9: Comparison image of coastlines in adjacent years: (a) 1990 and 2000, (b) 2000 and 2010, and (c) 2010 and 2018 and in four years.

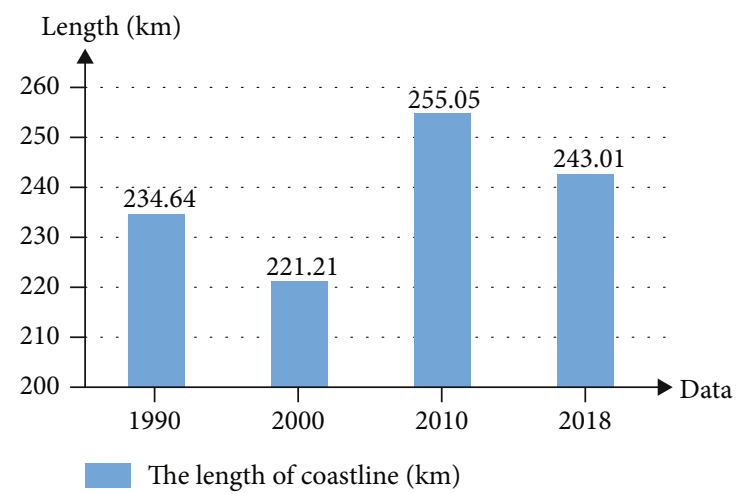

FIGURE 10: Length of Jiaozhou Bay coastline in each year.

the rivers not to disperse after entering the beach ponds. Accordingly, the sediment mainly accumulates in the sea area near the estuary, resulting in a shallow water depth. Sea reclamation is due to the rapid development of coastal factories before and after the founding of China and the reform and opening up. Sea reclamation is arbitrarily expanding, and the coastline is constantly advancing into the sea. This chain change caused the east coast coastline and the tidal flat to rise. The accumulation of municipal waste is also an important reason for the reduction in the area of Jiaozhou Bay. Besides the above-mentioned reasons, a substantial amount of garbage is piled up due to its proximity to the urban area on the east coast. This condition results in a shallow sea water depth and destruction of the original intertidal ecosystem.

\section{Conclusions}

This study described the theoretical basis, implementation process, and performance of a general coastline extraction method by using an improved active contour model. Firstly, MNDWI was applied to images to obtain water information based on the green and middle-infrared band information. Secondly, the improved active contour model was used to extract the coarse water area. Thirdly, the coarse water areas were processed by mathematical morphology to generate the seawater area, and the general coastline was extracted. Finally, the coastline was refined to remove the impacts of mathematical morphology processing. Landsat images over Jiaozhou Bay in Shandong Province, China, from 1990 to 2018 were used to extract the general coastline. The results 
demonstrate that the proposed method can effectively extract the general coastline, which is close to the reference coastline, especially for areas of land cover changes.

The changes in the length of the coastline in Jiaozhou Bay during 1990 to 2018 were analysed. The total length of the coastline had been increasing from $234.64 \mathrm{~km}$ in 1990 to $243.01 \mathrm{~km}$ in 2018 . However, this change has a significant increase by $33.84 \mathrm{~km}$ from 2000 to 2010 . The main reason is that Hongdao Island was integrated with the mainland because of the land reclamation. Thereafter, the length of the coastline slowly decreased by approximately $12 \mathrm{~km}$ from 2010 to 2018 due to the protective measures to the environment and reclamation prohibition.

In conclusion, the proposed method can effectively extract the general coastline from a remote sensing image, especially for human coastline. This improvement is beneficial for monitoring coastline change and marine ecological environment.

\section{Data Availability}

The data used to support the findings of the study are available from the corresponding author upon request.

\section{Conflicts of Interest}

The authors declare that there is no conflict of interest regarding the publication of this paper.

\section{Acknowledgments}

The authors acknowledge and appreciate the support of the Fundamental Research Funds for the Central Universities under Grant 2021 YCPY0113 and a project funded by the Priority Academic Program Development of Jiangsu Higher Education Institutions.

\section{References}

[1] C. Kuenzer, M. Ottinger, G. Liu, B. Sun, R. Baumhauer, and S. Dech, "Earth observation-based coastal zone monitoring of the Yellow River Delta: dynamics in China's second largest oil producing region over four decades," Applied Geography, vol. 55, pp. 92-107, 2014.

[2] W. Li and P. Gong, "Continuous monitoring of coastline dynamics in western Florida with a 30-year time series of Landsat imagery," Remote Sensing of Environment, vol. 179, pp. 196-209, 2016.

[3] H. Wu, Z. Li, K. C. Clarke et al., "Examining the sensitivity of spatial scale in cellular automata Markov chain simulation of land use change," International Journal of Geographical Information Science, vol. 33, no. 5, pp. 1040-1061, 2019.

[4] P. Maglione, C. Parente, and A. Vallario, "Coastline extraction using high resolution WorldView-2 satellite imagery," European Journal of Remote Sensing, vol. 47, no. 1, pp. 685-699, 2014.

[5] C. Dai, I. M. Howat, E. Larour, and E. Husby, "Coastline extraction from repeat high resolution satellite imagery," Remote Sensing of Environment, vol. 229, pp. 260-270, 2019.
[6] Z. Liu, F. Li, N. Li, R. Wang, and H. Zhang, "A novel regionmerging approach for coastline extraction from sentinel-1A IW mode SAR imagery," IEEE Geoscience and Remote Sensing Letters, vol. 13, no. 3, pp. 1-5, 2016.

[7] D. C. Mason and I. J. Davenport, "Accurate and efficient determination of the shoreline in ERS-1 SAR images," IEEE Transactions on Geoscience and Remote Sensing, vol. 34, no. 5, pp. 1243-1253, 1996.

[8] X. Liu, R. Jia, Q. Liu, C. Y. Zhao, and H. M. Sun, "Coastline extraction method based on convolutional neural networks-a case study of Jiaozhou Bay in Qingdao, China," IEEE Access, vol. 7, pp. 180281-180291, 2019.

[9] D. Cheng, G. Meng, G. Cheng, and C. Pan, "SeNet: structured edge network for sea-land segmentation," IEEE Geoscience and Remote Sensing Letters, vol. 14, no. 2, pp. 247-251, 2017.

[10] M. Silveira and S. Heleno, "Separation between water and land in SAR images using region-based level sets," IEEE Geoscience and Remote Sensing Letters, vol. 6, no. 3, pp. 471-475, 2009.

[11] T. F. Chan and L. A. Vese, "Active contours without edges," IEEE Transactions on Image Processing, vol. 10, no. 2, pp. 266-277, 2001.

[12] Z. Zhu, Y. Tang, J. Hu, and M. An, "Coastline extraction from high-resolution multispectral images by integrating prior edge information with active contour model," IEEE Journal of Selected Topics in Applied Earth Observations and Remote Sensing, vol. 12, no. 10, pp. 4099-4109, 2019.

[13] V. Caselles, R. Kimmel, and G. Sapiro, "Geodesic active contours," International Journal of Computer Vision, vol. 22, no. 1, pp. 61-79, 1997.

[14] X. Shi, C. Zhu, X. Ding, Q. du, N. H. Younan, and L. Li, “A superpixel-based coastline extraction algorithm for singlepolarized ENVISAT and ERS imagery," IEEE Journal of Selected Topics in Applied Earth Observations and Remote Sensing, vol. 12, no. 12, pp. 5118-5133, 2019.

[15] A. Fisher, N. Flood, and T. Danaher, "Comparing Landsat water index methods for automated water classification in eastern Australia," Remote Sensing of Environment, vol. 175, pp. 167-182, 2016.

[16] N. Otsu, "A threshold selection method from gray-level histograms," IEEE Transactions on Systems Man \& Cybernetics, vol. 9, no. 1, pp. 62-66, 1979.

[17] M. Hao, W. Shi, H. Zhang, and C. Li, "Unsupervised change detection with expectation-maximization-based level set," IEEE Geoscience and Remote Sensing Letters, vol. 11, no. 1, pp. 210-214, 2014.

[18] S. Ahmadi, M. J. V. Zoej, H. Ebadi, H. A. Moghaddam, and A. Mohammadzadeh, "Automatic urban building boundary extraction from high resolution aerial images using an innovative model of active contours," International Journal of Applied Earth Observation and Geoinformation, vol. 12, no. 3, pp. 150$157,2010$.

[19] S. Osher and J. A. Sethian, "Fronts propagating with curvature-dependent speed: algorithms based on HamiltonJacobi formulations," Journal of Computational Physics, vol. 79, no. 1, pp. 12-49, 1988.

[20] Y. Bazi, F. Melgani, and H. D. Al-Sharari, "Unsupervised change detection in multispectral remotely sensed imagery with level set methods," IEEE Transactions on Geoscience and Remote Sensing, vol. 48, no. 8, pp. 3178-3187, 2010. 
[21] S. Drazic, "Advanced morphological distances based on dilation and erosion," Fundamenta Informaticae, vol. 164, no. 1, pp. 17-39, 2019.

[22] Y. Zhang, R. Tong, D. Song, X. Yan, L. Lin, and J. Wu, “Joined fragment segmentation for fractured bones using GPUaccelerated shape-preserving erosion and dilation," Medical \& Biological Engineering \& Computing, vol. 58, no. 1, pp. 155-170, 2020. 